\title{
La place de la recherche dans le développement professionnel des formateurs en IUFM
}

\section{Stéphane Brau-Antony}

\section{(2) OpenEdition}

1 Journals

\section{Édition électronique}

URL : http://journals.openedition.org/trema/2930

DOI : 10.4000/trema.2930

ISSN : 2107-0997

\section{Éditeur}

Faculté d'Éducation de l'université de Montpellier

\section{Édition imprimée}

Date de publication : 1 juin 2013

Pagination : $22-35$

ISBN : 1167-315X

ISSN : 1167-315X

\section{Référence électronique}

Stéphane Brau-Antony, "La place de la recherche dans le développement professionnel des formateurs en IUFM », Tréma [En ligne], 39 | 2013, mis en ligne le 01 juin 2015, consulté le 19 avril 2019. URL : http://journals.openedition.org/trema/2930; DOI : 10.4000/trema.2930

Ce document a été généré automatiquement le 19 avril 2019

Trema 
La place de la recherche dans le développement professionnel des formateurs en IUFM

Stéphane Brau-Antony

I. Introduction 
1 Si depuis une quinzaine d'années le développement professionnel des enseignants est appréhendé comme un objet de recherche à part entière (Uwamariya et Mukamurera, 2005), c'est un domaine encore peu exploré chez celles et ceux dont la mission consiste à former les enseignants. En effet, le développement professionnel entendu comme «un processus de transformations individuelles et collectives des compétences et des composantes identitaires mobilisées ou susceptibles d'être mobilisées dans des situations professionnelles » (Barbier, Chaix et Demailly, p. 7) reste très peu étudié du côté des formateurs d'enseignants même si la question de leur professionnalisation est parfois abordée

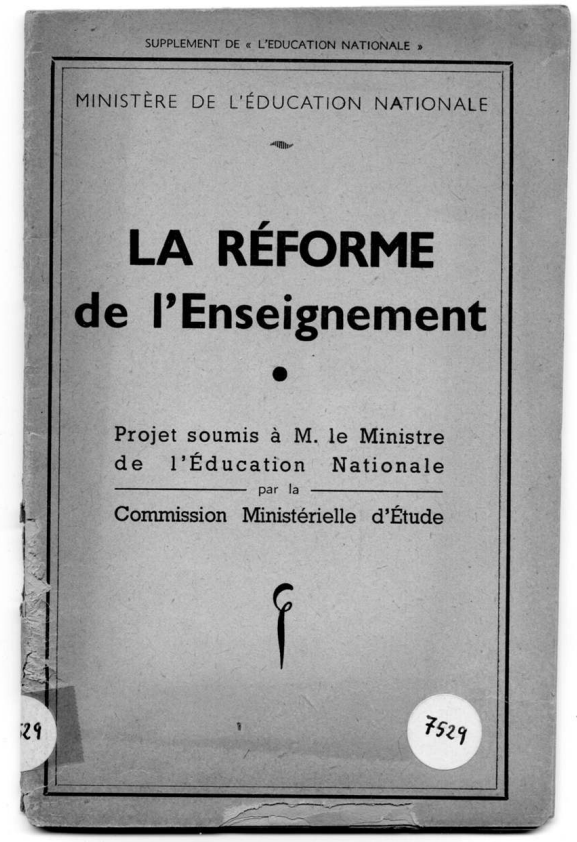
(Altet, Paquay et Perrenoud, 2002).

2 En IUFM les formateurs constituent une population très hétérogène. Ils peuvent être enseignant-chercheur (EC), enseignant du primaire ou du secondaire, à temps plein ou à temps partagé. Dégager ainsi les caractéristiques de la professionnalité d'un formateur d'enseignants est un redoutable défi tant les statuts, les tâches qui leur sont confiées et les identités diffèrent. Pour autant, ces formateurs mobilisent de véritables compétences pour exercer un métier dont les contours sont parfois difficiles à cerner car leur activité professionnelle est très composite. L'enquête menée par Altet (2002) souligne ainsi la grande diversité des tâches à accomplir telles que concevoir un programme de formation, faire de l'analyse de pratique, accompagner un stagiaire dans l'entrée dans le métier, diriger un mémoire ou un écrit professionnel ... Par ailleurs, les formateurs ${ }^{1}$ mettent en avant l'importance que peut jouer la recherche dans la formation des enseignants à condition, d'une part, qu'elle soit proche des situations professionnelles rencontrées par le praticien (« au plus près du terrain $»^{2}$ ) et, d'autre part, de «la rendre plus accessible». Certains formateurs revendiquent même un travail en partenariat avec les EC pour s'initier à la recherche : une façon comme une autre de signaler l'envie ou la nécessité de se former à et/ou par la recherche, cette activité restant étrangère à leur habitus professionnel étant donné leur statut.

Ces propos de formateurs mettent en évidence, premièrement, que la recherche semble avoir toute sa place dans la formation des enseignants mais cela suppose d'imaginer des modalités de rapprochement entre le monde de la pratique et celui de la recherche en ayant notamment accès à des travaux de recherche qui soient accessibles. Deuxièmement, certains formateurs se perçoivent comme étant capables de tisser des liens entre les savoirs issus de l'expérience et les savoirs issus de la recherche. Si tel est le cas, il convient d'élaborer des dispositifs de formation permettant aux formateurs de se former à une activité de recherche, la formation à et par la recherche étant considérée comme une voie possible de développement professionnel. 
4 Le développement professionnel se situe donc ici dans une perspective professionnalisante, il combine deux orientations :

- le développement professionnel s'inscrit dans le cadre de la formation continue ou de la formation de formateurs, il est considéré comme un apprentissage en tant que processus d'acquisition de savoirs professionnels provoquant des changements dans la façon d'exercer son métier (Day, 1999) ;

- le développement professionnel passe par la recherche et la pratique réflexive (Schön, 1994). Il s'agit d'être capable de rendre compte de sa pratique et de l'expliciter en utilisant des savoirs produits par la recherche, ces derniers sont conçus comme des grilles de lecture permettant de saisir la complexité des situations professionnelles dans toute leur singularité.

5 Après avoir évoqué la place que peut occuper la recherche dans le développement professionnel des formateurs nous présenterons dans cet article un dispositif de formation qui vise à former les formateurs à et par la recherche. Ce dispositif associe un EC et des formateurs. En préalable, nous poserons les conditions qui permettent selon nous de favoriser l'incorporation par les formateurs de savoirs issus de la recherche ce qui implique une formation de type méthodologique et l'appropriation de données théoriques. Nous exposerons dans un second temps le dispositif de formation en insistant sur la dimension méthodologique de la formation et en décrivant les principales étapes par lesquelles sont passés les participants. Nous chercherons à tracer le cheminement du groupe à travers les difficultés et obstacles rencontrés à la fois par les formateurs et l'EC. Enfin nous tenterons d'identifier les effets produits par ce dispositif du point de vue du développement professionnel des formateurs.

\section{Créer un espace de rencontre entre chercheurs et formateurs}

6 La formation à et par la recherche renvoie à la problématique de la circulation des savoirs entre recherche et formation et aux différentes façons d'organiser la rencontre entre praticiens et chercheurs. Proposer à des chercheurs de présenter leurs travaux en faisant l'hypothèse que le transfert va s'opérer dans les pratiques professionnelles des formateurs relève de la croyance naïve et de l'utopie (Huberman, Gather-Thurler, Nufer, 1998). Plusieurs conditions apparaissent indispensables afin que les travaux de recherche puissent constituer un instrument de travail (Perrenoud, Altet, Lessard, Paquay, 2008) pour les formateurs.

7 La première condition consiste à créer des espaces d'intéressement (Derouet, 2002) autour de préoccupations professionnelles partagées par les formateurs. Nous faisons l'hypothèse que l'articulation entre les savoirs issus de la recherche et ceux issus de l'expérience n'est possible qu'à partir du moment où elle s'appuie sur les situations habituelles de travail des formateurs (Brau-Antony 2009). "L'enrôlement» des formateurs est à ce prix afin d'éviter que se creuse un fossé entre deux cultures antagonistes : celle des professionnels de la formation et celles des professionnels de la recherche. Il est également important de souligner que l'investissement des formateurs ne peut s'effectuer que s'il existe une prise en charge institutionnelle. L'IUFM est-il prêt par exemple à s'engager dans la formation de formateurs? Quels sont les moyens financiers qui sont prévus ? Quel intérêt y a-t-il à mettre en place une action de formation 
à et par la recherche pour les formateurs alors qu'il y a d'autre lieux de formation possibles (master et doctorat)?

8 La seconde condition concerne le choix des acteurs les mieux placés pour jouer le rôle de «passeurs » et assurer la circulation des savoirs entre recherche et formation. Ceux qui apparaissent comme les plus légitimes sont évidemment les EC, toutefois on ne peut se contenter de l'évidence de la réponse. En premier lieu, il est indispensable que les objets de recherche sur lesquels travaille l'EC soient en adéquation avec les situations de travail des formateurs. À ce titre, les recherches en éducation et/ou sur la formation des enseignants sont à privilégier de façon à faciliter la collaboration formateurs/EC. Cependant affirmer que ces recherches sont les plus à même de trouver un écho chez les formateurs est insuffisant. Le travail de l'EC consiste à faire subir aux savoirs issus de la recherche un certain nombre de traitements afin que les formateurs puissent s'en emparer. Il convient d'après Martinand (2002) de reproblématiser ces savoirs afin qu'ils puissent constituer de véritables ressources pour les formateurs. Familiariser ces derniers avec les savoirs produits par la recherche nécessite donc, pour faire sens, que ces savoirs soient partiellement déconstruits puis reconstruits en fonction du milieu dans lequel ils seront mobilisés. En second lieu, une des difficultés majeures pour l'EC réside dans la position d'intériorité/extériorité qu'il occupe au sein du dispositif. Il doit ainsi être capable de gérer des tensions qui renvoient à son double statut mais aussi à une double identité, celle de chercheur garant d'une certaine rigueur méthodologique et celle de formateur animant le groupe de formation. Le « cheminement éthique» (Laplante, 2005) du chercheur engagé dans ce type de formation est tortueux, complexe et parfois déstabilisant. Sa mission première n'est pas de produire des connaissances dans un champ déterminé (visée heuristique) mais de concevoir et mettre en œuvre un dispositif au service du développement professionnel des formateurs (visée praxéologique).

\section{Un dispositif de formation original : le Groupe de Recherche Formation}

9 Le Groupe de Recherche Formation (GRF) que nous évoquerons dans cet article a pour objectif de "permettre à des formateurs non universitaires de participer à une activité de recherche et de s'approprier cette démarche » (extrait du plan de formation de formateurs). S'inscrivant dans la durée (2 ou 3 ans), le GRF associe des formateurs et un EC, celui-ci assumant le pilotage du dispositif de formation. Dans le cadre de cet article nous rendrons compte d'un dispositif de formation portant sur le conseil pédagogique d'enseignants stagiaires du secondaire. Ce dispositif se rapproche de celui conçu par Loizon (2008) pour des formateurs de l'enseignement primaire.

\section{1. Objectifs visés et déroulement de la formation}

Comme nous l'indiquions précédemment, le point de départ a consisté à mobiliser les formateurs autour de préoccupations professionnelles communes. Le choix de travailler sur le conseil pédagogique s'est rapidement imposé. En effet les formateurs avaient pour mission d'accompagner les stagiaires dans l'entrée dans le métier et à ce titre la visite du stagiaire sur son lieu de travail constituait un des moments clés de la formation. Nous sommes partis de l'hypothèse que l'étude approfondie de l'activité d'un conseiller pédagogique $(\mathrm{CP})$ lorsqu'il observe son stagiaire quand il fait la classe et mène ensuite un 
entretien post-séance était une façon d'interroger sa pratique de formateur lorsque l'on fait soi-même une visite de stagiaire. Le regard détaillé sur la pratique d'un pair favoriserait ainsi un retour réflexif sur sa propre activité par le recours à des savoirs produits par la recherche. Ce dispositif proche de ce que Barbier et Galatanu (2004, p.66) appellent une recherche-formation avait pour but de «faire produire les savoirs par les acteurs mêmes qui sont censés les détenir et les mobiliser » afin d'améliorer leur compétence à mener un entretien-conseil.

116 formateurs (anglais, EPS, mathématiques) et un EC en sciences de l'éducation ont participé à ce GRF, l'EC assumant lui aussi des fonctions de conseil au stagiaire. Le dispositif s'est étalé sur 3 ans avec 4 journées de regroupement par an et un travail intersessions consacré au recueil et au traitement des données. Chaque journée de regroupement a fait l'objet d'un compte-rendu rédigé par un des formateurs et soumis à discussion la journée suivante.

\section{2. L'inscription des participants dans une démarche de recherche}

\section{2. 1. Définir l'objet d'étude}

12 Nous souhaitions explorer l'activité d'un $\mathrm{CP}$ afin d'identifier ce qui se passe et ce qui se joue entre un $\mathrm{CP}$ et son stagiaire lors d'une visite. Cerner l'objet d'étude fut un redoutable défi, tâche en effet délicate pour des formateurs peu habitués à ce type de réflexion. Prendre conscience que la stabilisation de l'objet d'étude est une phase importante dans une démarche de recherche ne va pas de soi. C'est ce qu'atteste cet extrait de compterendu : « est-ce qu'on se contente d'étudier le contenu du discours entre le CP et son stagiaire lors de l'entretien post-séance par exemple ce que dit le CP (les aspects didactiques, la gestion de classe) ou est-ce qu'on s'intéresse à la dynamique des échanges entre les deux? On sait que la relation entre les deux est dissymétrique étant donné que c'est le $\mathrm{CP}$ qui a le pouvoir de juger et de décider si ça convient ou pas ». Ce propos nous semble significatif de la difficulté à bien délimiter ce sur quoi devait porter l'investigation. Dans le cas présent, il ne s'agissait pas d'étudier uniquement l'activité du CP du point de vue du contenu du discours qu'il tient au stagiaire, par exemple ses conseils concernent-ils en priorité ce qui est enseigné ou les situations d'apprentissage proposées aux élèves, son évaluation a-t-elle pour objectif de pointer des manques au niveau du contrôle de la classe?

Cet autre extrait issu d'un rapport d'activité réalisé au bout d'une année par l'EC fait état d'un recadrage qui a pour objectif de cerner l'objet de recherche et de convoquer les cadres théoriques qui s'y rapportent: «la situation de formation (l'entretien post-séance) relève avant toute chose d'une situation d'interactions entre deux protagonistes, cette situation pouvant être définie:

- Comme un processus d'influences mutuelles qu'exercent ou tentent d'exercer l'un sur l'autre les deux participants à l'interaction;

- comme le lieu où se joue cette interaction.

On parlera ainsi de lieu de rencontre dans le sens où l'interaction suppose de prendre en compte l'ensemble des événements qui composent cette interaction: par exemple ses différentes unités constitutives: séquences conversationnelles et types d'interactions (Kerbrat-Orecchioni, 1980).

Par ailleurs, il est important de préciser que ces échanges entre le CP et le stagiaire s'inscrivent dans un cadre participatif (Goffman, 1987) qui permet de caractériser la situation de communication et de donner du sens aux interactions que nous allons étudier. Le cadre participatif 
définit ainsi des éléments de contexte qui sont à la fois préétablis et co-élaborés au fur et à mesure $d u$ déroulement de l'interaction. Ce contexte peut être abordé de façon macro, méso et microscopique:

- Le contexte macro concerne le contexte institutionnel et social général de la situation de communication (formation en alternance, missions d'accompagnement du CP ...) ;

- le contexte méso concerne le cadre spatio-temporel et la situation sociale locale dans lesquels s'inscrit l'interaction entre le CP et le stagiaire, le nombre de protagonistes de la situation d'échange, leurs statuts et rôles, la nature des relations entre eux, les règles qui régissent les interactions et l'activité en jeu (dimension contractuelle...) ;

- le contexte micro concerne le micro-système individu avec ses caractéristiques propres. Nous avons affaire ici à des individus en activité qui lorsqu'ils s'engagent dans la situation d'interaction, le font avec leurs propres motifs en mobilisant leurs ressources cognitives, attentionnelles et émotionnelles ».

L'objet d'étude a pu ainsi être formulé de la manière suivante: « décrire et comprendre la dynamique des interactions entre un CP et son stagiaire lors d'un entretien-conseil » (extrait de compte-rendu).

\section{2. 2. Prélever et traiter les données}

\section{2. 2. 1. Le recueil de données}

Deux types de données ont été prélevés :

A/ un enregistrement audio-vidéo d'une séance d'anglais réalisée par un stagiaire avec une classe de $4 \mathrm{e}$ au premier trimestre. Nous disposions également des traces écrites concernant la préparation de la séance. Les objectifs linguistiques portaient sur la " capacité à décrire une scène dans le passé et de parler des événements qui sont survenus à un moment précis du passé (fonction langagière) et sur la capacité à distinguer et utiliser le prétérit simple et le prétérit Be-ing (structures langagières) ${ }^{3}$ ». La séance a été filmée en plan large de façon à pouvoir suivre les déplacements du stagiaire ainsi que l'ensemble des élèves de la classe ;

$\mathrm{B} /$ un enregistrement audio-vidéo de l'entretien post-séance entre le $\mathrm{CP}^{4}$ et son stagiaire qui a eu lieu immédiatement après la séance.

Les formateurs ont collecté l'ensemble des données et transcrit verbatim l'enregistrement audio-vidéo de l'entretien post-séance.

\section{2. 2. 2. Le traitement des données}

L'entretien post-séance a fait l'objet d'un premier type d'analyse, une analyse de contenu de type catégoriel (Bardin, 1991) qui a permis de diviser l'ensemble des communications émises par le $\mathrm{CP}$ et le stagiaire en trois composantes qui renvoient à une modélisation de l'activité enseignante empruntée à Doyle (1986) et Bru (1993 ) :

- la composante didactique relative aux savoirs enseignés et à l'activité de régulation de l'enseignant (situations d'apprentissage, choix de documents, correction des erreurs ...) ;

- la composante organisationnelle qui englobe la gestion de l'espace, l'organisation matérielle, la gestion du temps et des groupes ...;

- la composante relationnelle qui concerne les sollicitations de l'enseignant : feed-back, encouragements, rappels à l'ordre ou sanctions. 

individuellement puis nous avons confronté le codage de chaque participant. Afin de tester la validité des opérations de codage, nous avons considéré le codage comme fiable à partir du moment où 5 personnes sur 6 avaient codé de la même façon.

Nous avons cependant considéré cette première analyse comme insuffisante au regard de notre objet d'étude. En effet si le texte de l'entretien post-séance a un contenu manifeste, on ne pouvait accéder par la seule analyse de son contenu à sa signification. Il nous a donc fallu avoir recours à une théorie du langage. La dynamique des échanges a ainsi été étudiée en référence aux méthodologies d'analyse du discours selon un cadre inspiré des approches interactionnistes et des théories de l'énonciation (Charaudeau et Maingueneau, 2002). Dans cette perspective, le discours n'est plus considéré comme une simple transmission d'informations mais comme une forme d'action : "si parler, c'est sans doute échanger des informations, c'est aussi effectuer un acte régi par des règles précises, qui prétend transformer la situation de récepteur, et modifier son système de croyance et/ou son attitude comportementale; corrélativement comprendre un énoncé, c'est identifier, outre son contenu informationnel, sa visée pragmatique, c'est-à-dire sa valeur et sa force illocutoire » (Kerbrat-Orecchioni, 1980, p. 185).

21 La nouvelle grille d'analyse a permis dans un premier temps d'identifier des actes de langage. Parler d'acte de langage suppose de distinguer l'acte par lequel on produit un énoncé, l'énoncé lui-même (" matériel » puisqu'on peut l'enregistrer), l'acte par lequel on le comprend, mais aussi l'énonciateur qui le produit, le ou les destinataires qui le comprennent. Nous avons ainsi repéré dans l'entretien post-séance un certain nombre d'actes de langage du CP en référence notamment aux travaux de Dugal (2009) :

- évaluer (porter un jugement de valeur sur les choix du stagiaire) ;

- questionner;

- soutenir ;

- prescrire (imposer une solution);

- conseiller, suggérer, proposer des pistes ;

- reformuler/synthétiser certains propos du stagiaire pour recadrer l'entretien ;

- informer le stagiaire (apports de connaissances, relevé d'événements s'étant déroulés pendant la séance).

Dans un second temps nous avons déterminé un certain nombre de positions énonciatives. Le langage en tant que moyen d'interaction fait apparaître des jeux d'influence et de pouvoir. Les interlocuteurs sont de fait plus ou moins obligés de se positionner, d'adopter une ou des stratégies en fonction du déroulement de l'entretien. Nous inspirant des propositions d'Alin (1996), nous avons construit la typologie suivante (cf. tableau 1). 
Tableau 1 : typologie des positions énonciatives

\begin{tabular}{|c|c|}
\hline $\begin{array}{c}\text { Positions } \\
\text { énonciatives }\end{array}$ & Définitions \\
\hline Affrontement & $\begin{array}{l}\text { Attitude de contestation, de conflit (au sens positif du terme) où } \\
\text { l'enjeu peut être un enjeu de concurrence et de valorisation des } \\
\text { savoirs du stagiaire ou du CP. Elle est liée aux énoncés qui } \\
\text { précèdent, l'énoncé peut être considéré comme relevant d'une } \\
\text { position d'affrontement s'il va dans un sens opposé à l'énoncé } \\
\text { auquel il réagit. }\end{array}$ \\
\hline Confirmation & $\begin{array}{l}\text { Expression d'une adhésion au contenu des énoncés qui peut être } \\
\text { considérée soit comme un ralliement soit comme une validation de } \\
\text { ses idées et/ou de ses pratiques. Elle est liée aux énoncés qui } \\
\text { précèdent et l'énoncé peut être considéré comme relevant d'une } \\
\text { position de confirmation s'il va dans le même sens que l'énoncé } \\
\text { auquel il réagit. }\end{array}$ \\
\hline Écoute & $\begin{array}{l}\text { Elle peut être initiatrice d'une phase de questionnement ou de } \\
\text { réorientation de ce questionnement ou du débat ; elle peut } \\
\text { également simplement faciliter l'expression de son interlocuteur. En } \\
\text { ce sens, ces deux attitudes ne constituent pas forcément une } \\
\text { réponse au contenu d'un énoncé précédent mais souvent le point } \\
\text { de départ de l'échange ou d'une nouvelle phase de l'échange. Elle } \\
\text { peut enfin vouloir signifier à son interlocuteur qu'en dépit de } \\
\text { l'absence de prises de positions explicites le locuteur est attentif, } \\
\text { intéressé par ce qui se passe dans les échanges. }\end{array}$ \\
\hline Évitement & $\begin{array}{l}\text { Elle consiste le plus souvent en une relativisation du problème ou en } \\
\text { un déplacement du débat sur un champ non abordé jusqu'ici. }\end{array}$ \\
\hline Inhibition & $\begin{array}{l}\text { Attitude involontaire qui place le locuteur dans l'impossibilité } \\
\text { d'adopter une autre position énonciative. }\end{array}$ \\
\hline $\begin{array}{l}\text { Laisser } \\
\text { faire }\end{array}$ & $\begin{array}{l}\text { Attitude volontaire d'attente qui combine paradoxalement les } \\
\text { postions énonciatives d'affrontement ou de confirmation avec celle } \\
\text { d'évitement. Stratégies privilégiées : attente, non intervention, } \\
\text { ignorance. }\end{array}$ \\
\hline Conformisation & $\begin{array}{l}\text { Se mettre en conformité avec la perception que le stagiaire a des } \\
\text { attentes du CP. }\end{array}$ \\
\hline
\end{tabular}

Enfin il a fallu découper le corpus en différentes séquences caractérisées par une forte cohérence thématique, chacune de ces séquences a été balisée en numérotant les tours de parole (cf tableau 2).

Tableau 2 : exemple de séquençage de l'entretien post-séance

\section{Séquence 3 :Tours de parole 33 à 73}

La séance

*Rappel des objectifs linguistiques grammaticaux / lexicaux

Les écarts entre prévu / réalisé : quelles sont les causes?

* Le problème lexical :

- vocabulaire à réactiver et/ou à élucider

- importance de l'apport lexical

- nécessité d'une aide lexicale pour certains.

*Répercussions sur le déroulement des activités / sur la participation des élèves / sur la gestion du temps.

C'est ce deuxième type d'analyse qui a fait surgir le plus de difficultés chez les formateurs. Tout d'abord difficulté à suspendre son jugement c'est-à-dire éviter de porter un jugement de valeur sur la façon dont le CP conduisait l'entretien avec le stagiaire. Il a ainsi fallu rompre avec la posture d'évaluateur et endosser celle du chercheur qui tente de construire des outils d'intelligibilité de la réalité qui se présente à lui, travail d'autant plus délicat que les formateurs exercent la même activité que celle du CP observé. Le rôle de l'EC est ici fondamental, afin que les formateurs changent de positionnement. Comme 
le souligne Barbier (2008, p. 138) la démarche de recherche implique de "produire d'autres significations que celles que les acteurs accordent spontanément à leurs actes, même si cette formule n'est pas complète elle a l'avantage d'insister sur le fait que les catégories mentales utilisées comme outils d'analyse et d'interprétation dans le travail de recherche ne sont pas celles qui sont spontanément utilisées par les acteurs ».

Le second type de difficulté renvoyait à l'appropriation d'un champ conceptuel que la plupart des formateurs découvrait, ce qui a nécessité de la part de l'EC la recherche de textes accessibles afin que les formateurs puissent se familiariser avec les concepts d'actes de langage et de positions énonciatives. Le rapport d'activité cité précédemment (partie III. 2. 1.) avait ainsi pour fonction non seulement de clarifier l'objet d'étude mais aussi de préciser le paradigme de recherche dans lequel était envisagé l'analyse des interactions $\mathrm{CP} /$ stagiaire au cours de l'entretien post-séance. Le travail de l'EC nous semble ici crucial, la phase de reproblématisation des savoirs produits par la recherche évoquée plus haut prenant dès lors tout son sens. Se contenter de mettre à disposition des formateurs des textes de recherche est loin de suffire. Il nous est apparu essentiel que ces textes soient confrontés en permanence aux données empiriques afin de les faire fonctionner comme grille d'analyse des échanges $\mathrm{CP} /$ stagiaire. C'est ainsi que les grilles d'analyse de Dugal (2009) et Alin (1996) ont pu être discutées puis remaniées en fonction des caractéristiques du corpus. Les actes de langages informer le stagiaire et reformuler/ synthétiser et la position énonciative conformisation ne figurant pas dans les travaux des deux auteurs ont été ajoutées afin de mieux décrire et comprendre le sens des propos du $\mathrm{CP}$ et du stagiaire.

Nous avons fait le choix dans cet article de ne pas faire une présentation détaillée des résultats. Si le travail effectué dans le GRF a permis en codant la totalité des interactions $\mathrm{CP} /$ stagiaire de saisir la dynamique des échanges et de cibler le contenu des propos des deux protagonistes, rappelons que les objectifs du dispositif mettaient l'accent sur la formation méthodologique (analyse de contenu et analyse de discours) en utilisant sur le plan conceptuel les apports des approches interactionnistes et des théories de l'énonciation. Nous signalerons simplement que l'entretien post/séance privilégie la dimension didactique: objectifs visés, choix des documents et régulation des apprentissages. Selon les moments de l'entretien les actes de langage du CP sont de l'ordre de l'évaluation, du conseil et du questionnement. Les positions énonciatives se situent dans les registres de l'écoute, de la confirmation et de la conformisation, ces deux dernières étant révélatrices d'une asymétrie de position vis-à-vis de l'objet d'interlocution c'est-à-dire la pratique professionnelle ; l'expérience du CP faisant face à l'inexpérience du stagiaire (Vinatier, 2007).

\section{Effets de formation perçus par les formateurs}

La rédaction par chaque formateur d'une production écrite nous a paru être un excellent exercice devant permettre à chacun, non seulement de relater son expérience, mais surtout de conceptualiser et d'analyser le travail réalisé au sein du GRF. La consigne qui leur était donnée était d'identifier les apports du GRF sur le plan professionnel. Nous avons collectivement effectué une analyse de contenu des textes au cours de la dernière journée de formation. Le but était de caractériser les différents effets de formation perçus par les formateurs afin de vérifier si un processus de développement professionnel s'était enclenché. 
Nous retiendrons principalement deux effets de formation qui sont mis en exergue par tous les formateurs. Le premier a trait, pour reprendre une expression d'un formateur, "à la formation à l'activité de recherche " $^{5}$ Tous les formateurs insistent sur :

A/ l'effort intellectuel, peu habituel et parfois semé d'embûches qui vise à circonscrire l'objet d'étude : «je fus également étonnée de constater que l'objet de recherche n'est pas figé une fois pour toute au début de la recherche, qu'il évolue, se transforme, se déconstruit, semble même parfois échapper aux membres du groupe au gré des questionnements, de l'avancée de la réflexion, des renoncements et des apports théoriques pour se fixer enfin ";

B/ l'appropriation de cadres théoriques divers permettant de lire autrement la situation d'entretien/conseil alors qu'elle leur est familière « en adoptant une vision plus externe et en s'extrayant de mon rôle de formatrice en anglais et de conseillère pédagogique ». Un formateur parle « d'indicateur de développement » qu'il attribue à l'incorporation de savoirs théoriques nouveaux tels ceux issus de la pragmatique interactionniste alors qu'un autre met en avant «la nécessité de prendre en compte le cadre énonciatif et de renouer en quelque sorte avec des connaissances anciennes en partie oubliées ». Celui-ci estime qu'elles se sont avérées pertinentes pour analyser ce qui se passe et ce qui se joue dans les interactions CP/ stagiaire. Par ailleurs, les formateurs appartenant à des disciplines différentes, « il a fallu interroger la compatibilité de mon cadre didactique initial (EPS) avec les spécificités des collègues des autres disciplines ». Cette remarque est à mettre en relation avec le premier travail de codage où la catégorie didactique fut l'objet de débat, selon les cultures disciplinaires la signification accordée à la notion de didactique ne permettait pas d'emblée de trouver un accord entre les participants (comment distinguer didactique et pédagogie). Un des formateurs mentionne l'intérêt de ces moments de confrontation, de controverses, de mise à l'épreuve de ses propres cadres de référence en soulignant que «l'influence $d u$ collectif sur le processus de développement m'est toujours apparue comme évidente et fondamentale ». Tous les formateurs citent d'ailleurs le travail en équipe comme un moteur de développement ;

$\mathrm{C} / \mathrm{le}$ fait «qu'une des exigences fondamentales du travail de recherche est la rigueur intellectuelle ", cette exigence passe par un travail de prélèvement et de traitement des données perçu comme très coûteux mais qui fait pendre conscience de la temporalité de l'activité de recherche avec ses réussites et ses impasses. Les formateurs évoquent ainsi systématiquement dans leur écrit les caractéristiques de la démarche de recherche, les étapes qui la jalonnent et les obstacles qui surgissent : « la fixation des catégories d'analyse, la longue et parfois fastidieuse stabilisation des grilles de lecture nourrie régulièrement des apports théoriques ... »; « impression de ne faire que coder, décoder, recoder ... tricoter, détricoter pour sans arrêt affiner la lecture. Travail ressenti comme fastidieux voire barbant mais aussi ressenti au fur et à mesure comme une nécessité pour qu'il soit réellement un travail de recherche». Un formateur admet cependant que cette démarche fut trop pénible en faisant état de «la difficulté à m'investir dans le travail inter-sessions de codage de l'entretien lié à un isolement professionnel pour traiter les données ».

31 Le second effet de formation prend toute sa consistance à travers les termes utilisés par les formateurs pour qualifier les retombées du dispositif sur le plan de l'analyse de leur pratique professionnelle quand ils mènent un entretien post-séance: «prise de distance critique », «posture réflexive ", "questionnement sur sa pratique ». Ces termes témoignent du rôle de l'analyse réflexive pour transformer le sujet à condition que l'on soit capable de " l'instrumenter » pour décrire et comprendre les situations de travail : «l'analyse poussée de cet entretien est devenue pour moi un instrument de formation en ce sens qu'il m'a amené à 
transformer mon expérience et à interroger mes modes de raisonnement "; « décortiquer ainsi l'activité d'un $\mathrm{CP}$ ne peut que nous ramener à notre propre pratique». Comme le note un formateur « cette action de formation a déclenché un véritable effet miroir dont il est impossible de ne pas tenir compte lors de nos entretiens post-visite ", certains formateurs envisageant même des perspectives concrètes de transformation de leurs propres façons d'agir : «j'ai appris un certain nombre de choses notamment à me taire pour favoriser la parole de l'Autre »; « la typologie des actes de langage et des positions énonciatives me permet à la fois de m'autoinformer sur la qualité de mon conseil mais aussi de rester lucide sur les effets de cet acte professionnel ».

D'autres effets de formation sont également mis en avant, on pourrait les qualifier d'effets induits ou collatéraux car ils n'étaient pas directement ciblés par le dispositif GRF :

- conception d'actions de formation continue au conseil pédagogique à partir des matériaux et grilles d'analyse utilisées dans le GRF ;

- encadrement des écrits professionnels et mise en place de modules d'analyse de pratiques ;

- évaluation de « la qualité de l'entretien post-séance dans l'épreuve d'admission « critique de séance $»$ du CAFIPEMF ${ }^{6} »$.

\section{Conclusion}

Nous pensons à travers cet article avoir avancé des pistes montrant que la formation à l'activité de recherche contribue au développement professionnel des formateurs à condition de créer des espaces de rencontre entre formateurs et chercheur. Le dispositif que nous avons décrit répond à cette volonté de tisser des liens étroits entre recherche et formation. À ce titre, la perception par les formateurs des effets produits par l'implication dans un GRF met en évidence des retombées qui sont doubles :

- une transformation du regard portée sur la recherche à travers la confrontation aux différentes étapes qui caractérisent la démarche de recherche: désignation de l'objet d'étude, appropriation de savoirs produits par la recherche dans le domaine du conseil pédagogique, recueil et traitement des données. Cette formation à et par la recherche a toutefois laissé de côté les aspects relatifs à communication des résultats et notamment à l'écriture de recherche alors que cela constitue une des dimensions fondamentales du travail de recherche où l'on distingue classiquement la démarche de construction de la recherche (celle que nous avons privilégiée) et celle d'exposition de la recherche (communications dans des colloques ou journées d'étude, publications d'articles);

- une transformation du regard portée sur leur activité professionnelle quand ils font une visite de stagiaire: un regard plus réflexif, plus critique, plus distancié étayé par les cadres théoriques utilisés dans le dispositif ; transformation également favorisée par les discussions, débats et controverses au sein d'un groupe que l'on pourrait assimiler à une communauté scientifique élargie (Oddone, Rey et Briante, 1981) de par la présence d'un EC et d'un collectif de formateurs.

Cet article plaide donc pour la conception de ce type de dispositif de formation qui vaut, nous semble-t-il, autant pour les formateurs d'enseignants que pour les enseignants du primaire ou du secondaire dans le cadre de la formation initiale et/ou continue (Grosstephan et Brau-Antony, 2009). Soulignons enfin que si le développement professionnel vise à élargir le répertoire de compétences professionnelles du sujet, il interroge aussi l'identité du professionnel dans sa composante individuelle. « La conviction 
qu'un formateur impliqué dans la formation d'enseignants a le devoir de maintenir le lien avec la recherche» incite à s'engager dans un processus d'apprentissage et de recherche ou de réflexion sur sa pratique qui peut parfois déboucher sur un changement de statut ou de fonction (poursuite en doctorat comme ce fut le cas pour deux formateurs, un des formateurs venant d'être qualifié en tant que Maître de conférences).

La lecture hebdomadaire, Enseignement primaire, cours élémentaire et moyen, Dutilleul, J., Ramé, A., s.d., $12^{\mathrm{e}}$ édition, Librairie classique André, E. fils. Paris. Source : CEDRHE 5086.

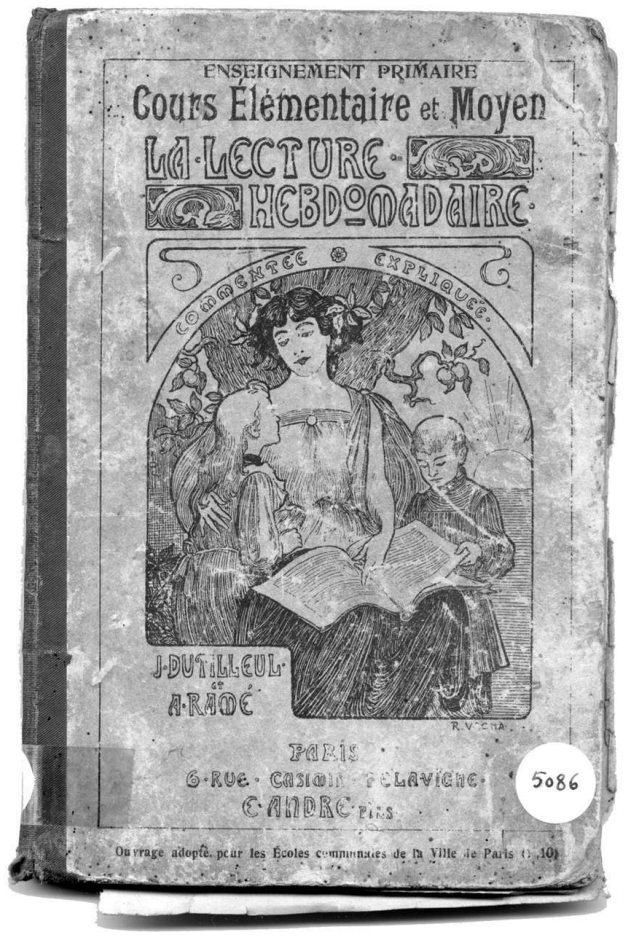

\section{BIBLIOGRAPHIE}

Alin, C., être formateur. Quand dire c'est écouter. Paris : L'Harmattan, 1996.

Altet, M., Quelle(s) professionnalités (s) des formateurs en formation continue ? Vers un profil polyidentitaire. In Altet, M., Paquay, L. et Perrenoud, P., Formateurs d'enseignants. Quelle professionnalisation? Bruxelles : De Boeck, 2002, p. 59-87.

Altet, M., Paquay L. et Perrenoud, P., Formateurs d'enseignants. Quelle professionnalisation? Bruxelles : De Boeck.

Barbier, J-M., Entretien avec F. Clerc. Recherche et Formation, 59, 2008, p.133-140.

Barbier, J-M., Chaix M-L. et Demailly, L., Éditorial Recherche et développement professionnel, Recherche et Formation, ${ }^{\circ} 17,1994$, p. 5-8. 
Barbier, J-M. et Galatanu, O., Savoirs, capacités, compétences, organisation des champs conceptuels. In J-M. Barbier et Galatanu, O., Les savoirs d'action : une mise en mots des compétences. Paris : L'Harmattan, 2004, p. 31-78.

Bardin, L., L'analyse de contenu. Paris : PUF, 1991.

Brau-Antony, S., Recherches en didactique et développement professionnel de formateurs d'enseignants, Savoirs, n²1, 2009, p. 132-147.

Bru, M., L'enseignant organisateur des conditions d'apprentissage. In. Houssaye, J., La pédagogie : une encyclopédie pour aujourd'hui. Paris : ESF, 1993, p. 103-117.

Charaudeau, P. et Maingueneau, D., Dictionnaire d'analyse du discours. Paris : Seuil., 2002.

Day, C., Developing teachers. The challenges of lifelong learning. Londres : Falmer Press, 1999.

Derouet, J-L., Du transfert à la circulation des savoirs et à la reproblématisation. De la circulation des savoirs à la construction d'un forum hybride et de pôles de compétences, Recherche et Formation. $n^{\circ} 40,2002$, p. 13-25.

Dugal, J-P. et Leziart, Y., La circulation des savoirs entre recherche et formation : l'exemple des concepts didactiques lors d'une action de formation de conseillers pédagogiques, Revue française de pédagogie, ${ }^{\circ} 146,2002$, p. 37-47.

Dugal, J.-P., Attitudes dans les entretiens conseil et formation des conseillers pédagogiques, Travail et formation en éducation, $\mathrm{n}^{\circ} 4,2009$, p. 1-18.

Doyle, W., Classroom knowledge as a foundation for teaching, Teacher College Records, $\mathrm{n}^{\circ} 91$, 1990, p. 347-360.

Goffman, E., Façons de parler. Paris : Les éditions de Minuit, 1987.

Grosstephan, V. et Brau-Antony, S., Le processus d'incorporation par des enseignants d'EPS de savoirs issus de la recherche. Exemple d'un dispositif de formation continue de type collaboratif associant un chercheur et des enseignants, eJRIEPS, $n^{\circ} 16,2009$, p. 22-37.

Huberman, M., Gather-Thurler, M. et Nufer, E., La mise en pratique des recherches scientifiques : étude de la dissémination des résultats du programme national de recherche : Éducation et vie active, Rapport exécutif. Genève : Université de Genève, Faculté de psychologie et des sciences de l'éducation, 1998.

Kerbrat-Orecchioni., L'énonciation. De la subjectivité dans le langage. Paris : Armand Colin, 1980.

Laplante, B., Cheminement éthique du chercheur engagé en recherche collaborative, Revue des sciences de l'éducation, $\mathrm{n}^{\circ} 31 / 2,2005$, p. 417-436.

Loizon, D., Former des conseillers pédagogiques du premier degré par la recherche, Recherche et Formation, n59, 2008, p. 105-119.

Martinand, J-L., Entretien avec E. Burguiere, Recherche et Formation, n40, 2002, p. 87-94.

Oddone, I., Rey, A. et Briante, G., Redécouvrir l'expérience ouvrière. Vers une autre psychologie du travail. Paris : Éditions sociales, 1981.

Schön, D-A., Le praticien réflexif. À la recherche du savoir caché dans l'agir professionnel. Montréal : Éditions Logiques, 1994.

Perrenoud, P., Altet, M., Lessard, C. et Paquay, L., Conflits de savoirs en formation des enseignants. Entre savoirs issus de la recherche et savoirs issus de l'expérience. Bruxelles : De Boeck, 2008. 
Uwamariya, A. \& Mukamurera, J., Le concept de « développement professionnel » en enseignement : approches théoriques, Revue des sciences de l'éducation, $n^{\circ} 31 / 1,2005$, p. 155-178.

Vinatier, I., Les enjeux de la dynamique intercationnelle entre conseillers et enseignants en formation et/ou débutants, Recherche en Éducation, $n^{\circ} 4,2007$, p. 65-76.

\section{NOTES}

1. Nous intéressant essentiellement dans cet article aux formateurs de statut secondaire (PRAG ou PRCE), le terme formateur ne s'appliquera pas au statut d'enseignant-chercheur.

2. En italique propos de formateurs tirés de l'enquête d'Altet (2002).

3. Extrait de la préparation de séance.

4. Le $\mathrm{CP}$ avait déjà exercé cette fonction plusieurs fois.

5. Les citations des formateurs sont écrites en italique.

6. Certificat d'Aptitude à la Fonction d'Instituteur, Professeur des Ecoles Maîtres Formateurs.

\section{RÉSUMÉS}

L'article met en évidence que la formation à l'activité de recherche peut contribuer au développement professionnel des formateurs à condition de créer un espace d'intéressement à partir de préoccupations professionnelles partagées par les formateurs. Sont décrits dans cet article le dispositif de formation à et par la recherche ainsi que les effets de formation perçus par les formateurs.

This article highlights that the training to research activity can contribute to trainers' professional development providing the creation of a space of concern/interest built from the professional preoccupations shared by the trainers. The training device to and by the research as well as the training effects perceive by the trainers are described in this article.

\section{INDEX}

Keywords : education, Professional development, research

Mots-clés : développement professionnel, formateur, recherche

\section{AUTEUR}

\section{STÉPHANE BRAU-ANTONY}

URCA / IUFM. MCF HDR 70e, CEREP (EA 4692) 